Research Article

\title{
Construction of a Hierarchical Neural Network Power Source Model for Human Capital Technology Innovation and Benefit Distribution with Big Data Analysis
}

\author{
Yang Liu ${ }^{1}{ }^{1}$ and Sang-Bing Tsai (iD ${ }^{2}$ \\ ${ }^{1}$ School of Economics and Management, Harbin University of Science and Technology, Harbin, Heilongjiang 150080, China \\ ${ }^{2}$ Regional Green Economy Development Research Center, School of Business, WUYI University, Nanping, Fujian, China
}

Correspondence should be addressed to Yang Liu; wlzy314215@126.com and Sang-Bing Tsai; sangbing@hotmail.com

Received 12 August 2021; Revised 31 August 2021; Accepted 9 September 2021; Published 17 September 2021

Academic Editor: Gengxin Sun

Copyright (c) 2021 Yang Liu and Sang-Bing Tsai. This is an open access article distributed under the Creative Commons Attribution License, which permits unrestricted use, distribution, and reproduction in any medium, provided the original work is properly cited.

\begin{abstract}
In this paper, a hierarchical neural network power source model is used to conduct an in-depth analysis and research on human capital technology innovation and revenue distribution. A hierarchical neural network analysis method was chosen to evaluate the human capital value of professional degree master students, and the applicability of the index system was confirmed through errors; moreover, the significance of the output results was analyzed according to the weight assignments of the input, implicit, and output layers. The analysis found that there was a large disagreement in the assessment of their human capital value, which led to the lack of practical utility of human capital. Knowledge-skilled talents have a wealth of theoretical knowledge and can use theories to guide related work. Compared with technically skilled high-skilled talents, their educational level is higher, and they can summarize past intuitive experience into theoretical guidance. Therefore, the hierarchical neural network method we constructed is theoretically effective in assessing the value of the human capital of professional master's students and the role of the main constituents. Based on the assessment results, we can provide policy-informed suggestions for improving the quality of school education. To quickly verify whether the model can converge during the training process, a simple dataset with only two sequences and the elements in the sequences being real numbers rather than vectors are constructed to speed up the computation; meanwhile, the length of the sequences in this dataset is adjustable to initially verify the model's ability to alleviate the long-time dependence problem.
\end{abstract}

\section{Introduction}

With the speed of information transmission and the spread of enterprise products in the world, consumers' expectation of product quality and service is getting higher and higher, and the enterprise competition front has been extended from product sales to the R\&D stage and economic globalization, which makes technological innovation more prominent in enterprise competition and becomes the core power of enterprises participating in international competition [1]. Highly skilled personnel are the front-line technicians who are active in various industrial production lines in Zhejiang Province, and they are the practitioners, operators, and converters of the results in the team of skilled workers, as well as the creators and designers of products involved in the production process [2]. The economic construction is undergoing the "cage for birds," and the industrial factor structure is changing from the "singlewheel drive" of the traditional processing manufacturing industry to the "double-wheel drive" of advanced manufacturing industry and modern service industry. Concerning transformation, these results are inseparable from the role of highly skilled personnel [3]. At present, the new production model of Zhejiang Province presents intelligent features, the emergence of this feature does not mean that it no longer needs highly skilled personnel to complete the corresponding front-line work, but the advanced manufacturing industry needs more technically 
talented people to play light and heat. The original traditional manufacturing industry requires the first-line highly skilled talents to only have skilled technical skills and be able to solve the problems encountered in the manufacturing process. But now, highly skilled personnel should not only master the technical skills required in the front-line work but also need the analysis and management ability of the intelligent operational system [4]. Borrowing the formula of talent flexibility, the quality of high-skilled talents in Zhejiang Province was predicted and analyzed. Finally, it puts forward opinions and suggestions on the cultivation of highskilled talents from the perspectives of educating, attracting, and retaining people. Highly skilled personnel, by renewing the technology and recreating the products, can drive the improvement of the total factor productivity of the enterprise and promote the acceleration of the pace of transformation and upgrading of the industrial structure.

Practice drives theory, and as competition intensifies, how to enhance the competitiveness of enterprises through effective HRM has become a common topic of concern for scholars. Traditionally, HRM is often assigned an operational role, and the focus of organizations on HRM is only on the smooth operation of daily operational activities such as recruitment, selection, training, performance evaluation, and compensation system. The study has not connected HRM activities with an organizational competitive advantage. To answer these questions, many scholars have looked at human capital and social capital to find answers [5]. Human capital is the idea that the stock of production knowledge, labor and management skills, and health qualities embedded in employees increases through the expenditure on education and vocational training of producers and the opportunity cost of receiving education, thus significantly improving and contributing to the technological innovation performance of firms; this is demonstrated by the correlation between investment expenditure on education and vocational training and the technological innovation performance of firms at the national and firm levels [6]. The impact of human capital on firms' innovation performance is reviewed through the correlation between country-level and firm-level investment in education and vocational training and firms' technological innovation performance. On the surface, this argument makes sense; however, as the research progresses, scholars find that employees with specific knowledge and skills are only a necessary but not a sufficient condition for improving the firm's technological innovation performance. Specifically, in terms of human capital investment, is there a problem of overinvestment in human capital to achieve technological innovation performance, i.e., if one unit of human capital investment costs two units of innovation performance, we need to consider whether there is a case where only 0.5 units of investment costs two units of innovation performance? Or is it possible to achieve 4 units of innovation performance? The key to real competitive advantage is the effectiveness of human capital. Comparing $\mathrm{Xu}$ Shu to the employees and Cao Cao to the enterprise, we can find that the role of human capital on the technological innovation performance of the enterprise is not sufficient, and we need to find another factor to give full play to the effectiveness of human capital.
The window slides over the sequence with a fixed stride length and ensures that the sliding of the window completely covers the entire input sequence, then each slide of the window produces a vector, and after the sliding of the window over the entire sequence is completed, a new sequence is generated. After the window slides over the whole sequence, a new sequence is generated, and this new sequence is guaranteed to be shorter than the original sequence, then a new windowed recurrent neural network slides over this new sequence, and so on until the length of the final sequence is within the tolerance range of the recurrent neural network, forming a hierarchical structure consisting of multiple recurrent neural networks. Second, in the recurrent neural network under the single output method, the layered sliding window recurrent neural network cannot converge in the training process, the full-sequence accumulation model is designed within the window, that is, all vector elements in the hidden layer sequence of the recurrent neural network in each window are summed up, but after the experiment, it is found that the full-sequence accumulation model still cannot converge. This paper combines the partial sequence output method of the recurrent neural network to construct the latter half. In this paper, we combine the partial sequence output of the recurrent neural network to build a post-half accumulation model, i.e., only the second half of the vector elements in the hidden layer of recurrent neural network in each window are added together, and it is proved that the layered sliding window recurrent neural network can converge after using the post-half accumulation model. Finally, considering that the difference between the full-sequence accumulation model and the post-half accumulation model is mainly the ratio of the accumulated vector elements to the vector elements of the whole window.

\section{Current Status of Research}

The research method of systems theory is used to analyze the dynamic evolutionary process of the economic system of human development. Widmer, the founder of systems science, and others generally believe that the system analysis approach has a large potential for development and a wide range of applications and can be fully applied to the field of economics to analyze problems and solve them [7]. In this paper, we analyze the evolution of the economic system of human development in depth by using the research method of the system science of the noncircle than the system, and at the same time, we elaborate the definition, characteristics, development laws, and dynamic mechanisms of the economic system of human development and construct the framework of the analysis of the system source of the source of human development and the analysis of the source of resistance and asymmetric information source of human development [8]. We divide this new sequence into several subsegments, then input the subsegments into the new recurrent neural network, and continue to deepen the network in this way. As the network deepens, the sequence keeps shortening until the length of the sequence reaches within the allowable range of the recurrent neural network. 
In addition, the system evolution dynamics model of human development is established, and technology and capital are analyzed as the main up and down factors of the economic system of human development, and the interactions and constraints of technology and capital in promoting human development are analyzed to integrate them, which can be regarded as a supplement and expansion of the economic theory of human development.

Baby, through interviews with 15 low-skilled adult workers in Italy, provides a corresponding view on the relationship between the opportunities for lifelong learning and structural constraints on participation in work and the personal strategies adopted by low-skilled workers in Italy, and he argues that the government should expand the opportunities for low-skilled workers to participate in learning and provide appropriate training [9]. Developed countries strongly encourage companies to build up their internal highly skilled workforce and to participate in the training of skilled personnel. Therefore, scholars have also studied the issue of apprenticeship and skills development [10]. Ghadiyaram investigated the operation of apprenticeships for low-skilled workers in Ghana by finding that there is a mismatch between apprenticeship training and industry conditions, lack of regulations, ineffective apprenticeship programs, insufficient investment in education and training, and outdated training programs in Ghana [11]. It is noteworthy that the requirements for the training of "low-skilled workers" abroad are not to train them to become "highly skilled" but partly to train them to become "middle-skilled." Zhou et al. argue that economic research should focus on the analysis of people's mutual exchange relations and then explain and anticipate the behavioral choices of people in the market exchange [12]. With the enrichment and improvement of economic theories, theories such as human capital theory, new development concept, and liberal development concept have started to emerge, and the core of these theories is the problem of human development, and they all attach great importance to the subjective role of people in development, which fully embodies the concept of people-oriented development [13]. The human capital theory emphasizes the important role played by human resources in economic growth and social progress and believes that human capital is more influential than physical capital, while human investment is an important means to improve the quality of the population and promote economic development. Ding and others pointed out that capable people are the key to modern economic abundance and that they are an important source of economic growth [14]. Migdał-Najman et al. also conducted relevant research on human development and concluded that the two main driving forces of human development are the pursuit of profit maximization and the pursuit of ethical perfection and that only by effectively integrating the two can we better promote economic development and social progress and then promote human development [15].

From the scholars' research on human development, no matter what kind of economic form, the research on human development is inseparable in the process of development. People are the main body of economic development, and only when the free and comprehensive development of people is the ultimate pursuit goal, economic development, and social progress will be scientific and sustainable. Knowledge-skilled talents have rich theoretical knowledge and can apply theories to guide relevant work. Compared with the technical-skilled high-skilled talents, their education level is higher and they can summarize their past intuitive experience into theoretical guidance. This type of talent not only has the characteristics of the first two types of highly skilled personnel but also has a certain degree of creativity and innovation. It is a person who can hold a certain management position in real work and can solve many positions and many technical level problems. Secondly, the current demand bottleneck and cultivation status of high-skilled talents are analyzed. In response to the above, through data mining and collation, a preliminary prediction of the demand for high-skilled talents in Zhejiang Province in the future period is made by using the hierarchical neural network prediction model, and the quality of high-skilled talents in Zhejiang Province is predicted and analyzed by borrowing the talent elasticity formula. Finally, opinions and suggestions are put forward for the cultivation of highskilled talents from the perspectives of cultivation, attraction, and retention.

\section{Hierarchical Neural Network Power Source Model Construction for Human Capital Technology Innovation Benefit Distribution Analysis}

3.1. Hierarchical Neural Network Power Source Model Design. Can the recurrent neural network also be organized into a larger network in this low-to-high manner? A very long sequence is divided into several small segments, each segment is input to the recurrent neural network to output a vector, and then, the output vectors corresponding to each segment form a new sequence, this new sequence is further divided into several small segments, and these new sequences divided into small segments are then input to the new recurrent neural network [16]. The solution in this article is to count the sequence lengths of all samples in the entire dataset, take the maximum sequence length, calculate the number of layers required for this maximum sequence length, and use this number as the total number of layers in the model. When the sample sequence length is relatively small, by adding a zero vector before the sequence, the sequence length of the current sample reaches the minimum sequence length required by the model. As the network deepens, the sequence is shortened until the length of the sequence reaches the range allowed by the recurrent neural network. The proposed hierarchical sliding window recurrent neural network is based on this idea, and the structure of the hierarchical sliding window recurrent neural network is shown in Figure 1.

The original RNN is simple in structure and convenient for theoretical analysis, so this chapter is used as the chapter of theoretical analysis, all formula derivation, diagrams, and text descriptions are used in the original RNN, but because 


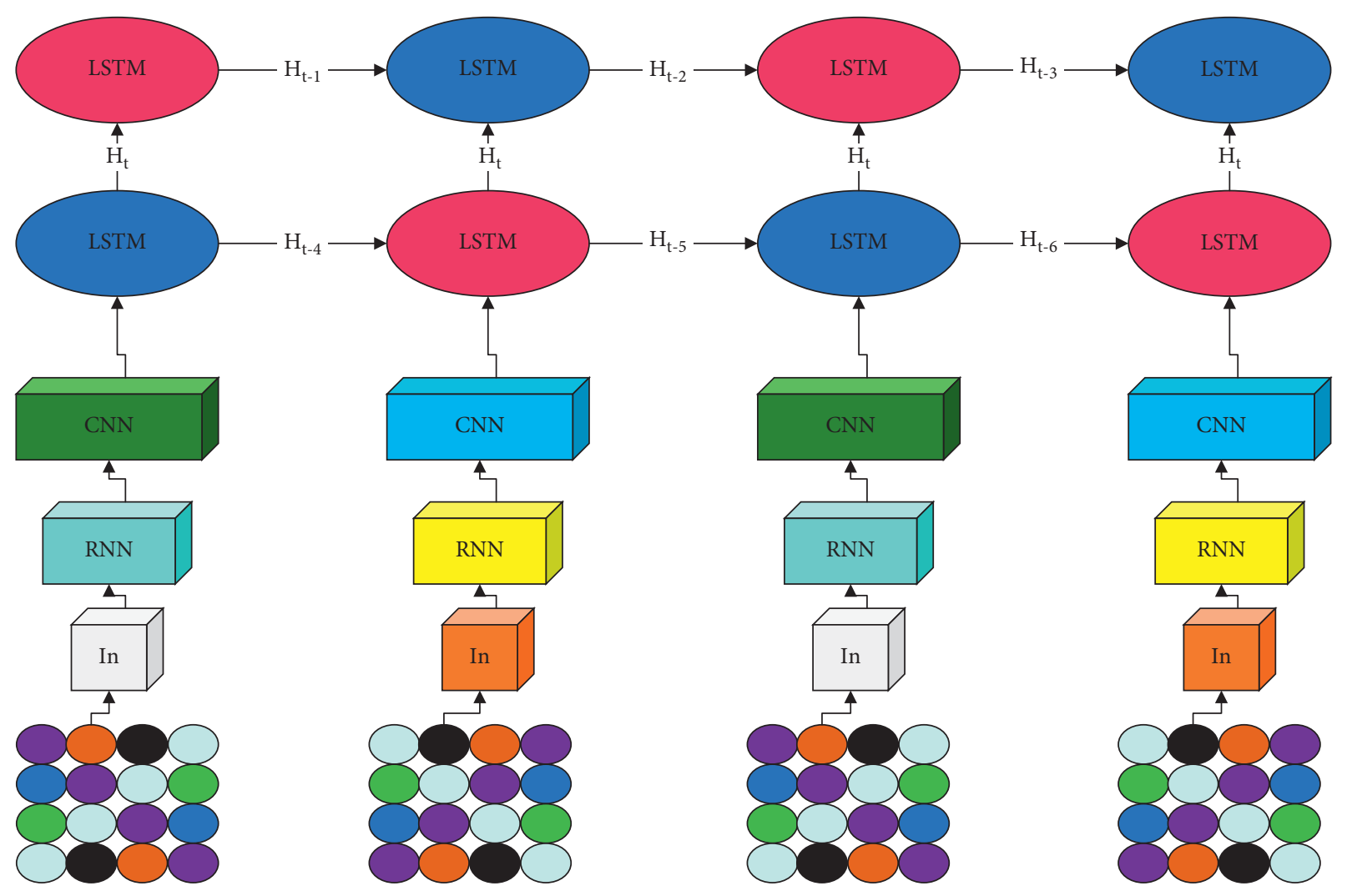

FIGURE 1: Layered sliding window recurrent neural network.

the function of the original RNN is too single, so the performance in practice is not satisfactory, so in the experimental stage of this paper, the recurrent neural network used complex and powerful LSTM or GRU across the step length of the sliding window recurrent neural network with an arbitrary step length of 1 . The mathematical expression of the layered sliding window recurrent neural network is as follows:

$$
\begin{aligned}
y_{k}^{L} & =h_{k, w}^{L}, \\
h_{k, w}^{L} & =f\left(U^{L} \cdot x_{s(k-1)+i}^{L}-V^{L} \cdot h_{k, j+1}^{L}\right), \\
x_{k}^{L} & =y_{k}^{L},
\end{aligned}
$$

where $y_{k}^{L}$ denotes the output vector of the $k$ th window in the $L$ th layer sliding window recurrent neural network, $h_{k, w}^{L}$ denotes the last vector in the sequence of the hidden layer of the $k$ th window in the $x_{s(k-1)+i}^{L}$ layer sliding window recurrent neural network, $w$ is the window width, $s$ is the sliding span length, $h_{k, w}^{L}$ denotes the input weight coefficient matrix in the $U^{L}$ layer sliding window recurrent neural network, VL denotes the hidden layer weight coefficient matrix in the $V^{L}$ layer sliding window recurrent neural network, $f(\cdot)$ is the activation function, and $x_{k}^{L}$ denotes the $j$ th vector of the input sequence in the $L$ th layer of the sliding window recurrent neural network, where all windows in the same layer share $U^{L}$ and $V^{L}$, and $U^{L} V^{L}$ in different layers are different.
In practice, the sequence length of samples usually varies, so there may be a situation where the input sequence length does not match the window width and stride length. In addition, the total number of layers in a hierarchical sliding window recurrent neural network is determined by the sequence length of the samples when the window width and stride length are fixed; that is, the larger the sequence length of the samples is, the more layers are needed [17]. When the sample sequence length is relatively small, the sequence length of the current sample is made to reach the minimum sequence length required by the model by adding zero vectors in front of the sequence.

Therefore, in this paper, the zero vectors are added in such a way where the zero vectors are added before the input sequence of each layer, the input sequence length after the zero vectors are added in the current layer is larger than or equal to the window width, and the input sequence length after the zero vectors are added in the current layer matches the window width and the stride length. This method makes the number of zero vectors added in each layer smaller than the window width, thus achieving a relatively small total number of zero vectors. In this paper, inspired by residual networks and ALSTM, we construct a full-sequence accumulation model within the window and sum up all vectors in the sequence of hidden layers of the recurrent neural network within a single window to obtain the output vector, and the mathematical expression is 


$$
y_{k}^{L}=\sum_{j=1}^{w}\left(h_{k, w}^{L}\right) .
$$

The design goal of a hierarchical sliding window recurrent neural network is to reduce the sequence length layer by layer; the greater the span length, the greater the reduction in sequence length layer by layer, but the greater the span length, the greater the possibility of losing more information. The packaging feature selection algorithm is not as common as the filtering feature selection algorithm, because if another classification algorithm is used, this method cannot guarantee that the selected feature is also the best feature for another classification algorithm. In addition, the packaging type feature selection algorithms are easier to overfit than filtered feature selection. Let the length of the output vector of the previous layer be $l$. The two adjacent vectors in the output sequence of the previous layer are merged into one vector, and the length of the merged vector is $2 l$. Then, the input vector of the current layer is obtained through a fully connected neural network shared by the current layer, and the vector length of the output vector of this fully connected layer is $l$, as shown in Figure 2.

The self-encoder can effectively act as a dimensionality reduction or sparse coding when the vector length of the implied layer of the self-encoder is smaller than the vector length of the input layer; the self-encoder acts as a dimensionality reduction; for example, a single layer fully connected self-encoder without activation function is equivalent to principal component analysis (PCA), which extracts the most valuable elements of the vector, so the fully connected dimensionality reduction layer can potentially reduce the information loss of information loss [18]. The mathematical expression of a layered sliding window recurrent neural network with a fully connected dimensionality reduction interval accumulation model is

$$
\begin{aligned}
y_{k}^{L} & =\sum_{j=1}^{w}\left(h_{k, 2 j+1}^{L}\right), \\
h_{k, 2 j+1}^{L} & =f\left(U^{L} \cdot x_{k+j+i}^{L}-V^{L} \cdot h_{k, 2 j+1}^{L}\right), \\
x_{k}^{L} & =f\left(W^{L} \cdot x_{k+j+i}^{L}-W^{L} \cdot h_{k, 2 j+1}^{L}\right) .
\end{aligned}
$$

Unlike filtered feature selection methods, packed feature selection methods use classification algorithms to evaluate feature subsets. Most of the time, the classification error rate or performance accuracy is used as a criterion for feature evaluation. It selects the most discriminative features by minimizing the prediction error. This approach usually achieves better performance than filtered feature selection methods because it considers feature dependencies and includes biases directly in the learning algorithm. However, wrapped feature selection algorithms are not as common as filtered feature selection algorithms. The hierarchical sliding window cyclic neural network is the model structure used in this paper to solve this problem, and the key to the work of the hierarchical sliding window cyclic neural network is to integrate the latter half accumulation model or interval accumulation model in it. If another classification algorithm is used, the method does not guarantee that the selected features are also the best features for the other classification algorithm, and wrapped feature selection algorithms are more prone to overfitting than filtered feature selection because the classification algorithm is used to compute each subset of features repeatedly. Most wrapped feature selection algorithms are multivariate, so they require many computational resources to achieve convergence and are also difficult to handle for large datasets, so they are also much less time-efficient than filtered feature selection algorithms.

We argue that employees' psychological capital affects corporate technological innovation performance, which in turn is influenced by the corporate innovation climate. Psychological capital and technological innovation performance are mainly expressed and perceived by employees themselves and thus can be categorized at the individual level, while innovation climate is perceived by individuals to the group and thus can be categorized at the group level. Due to the maturity of cross-level analysis techniques, researchers have gradually used cross-level analysis models to explore the influence of different factors on individual behavior, the most common of which is Hierarchical Linear Modeling (HLM). In this study, Hierarchical Linear Modeling is used to explore the influence of group factors (organizational innovation climate) and individual factors (employees' psychological capital) on the technological innovation performance of firms. Hierarchical linear model analysis (HLM) is a statistical analysis method developed when different levels of variables are considered simultaneously, and the biggest difference from the traditional analysis method is the treatment of different levels of variables. According to the suggestion of Hofmann and examining the hypotheses of this study (direct effect of Level 1, the direct effect of Level 2, moderating effect of Level 2), the analysis was performed in a cross-level analysis model.

$$
\begin{aligned}
k_{h} & =\sum_{m=1}^{M} v_{h m} \cdot z_{m}-b_{h}, \\
p_{h} & =f_{z p}\left(\beta_{h}, k_{h}\right)^{2}, \\
t_{e} & =f_{p t}\left(\sum_{m=1}^{M} v_{h m} \cdot z_{m}-b_{h} \beta_{h}, k_{h}\right) .
\end{aligned}
$$

It should be noted that to simplify the computation, $w_{e h}$ is randomly initialized to -1 or 1 , and the value is kept constant in subsequent training. This is done mainly for the following reasons: the connection matrix $v_{h m}$ from the input layer to the hidden layer and the connection matrix $w_{e h}$ from the hidden layer to the output layer can be considered as 2 lenses, and the output from the input layer to the hidden layer and the output from the hidden layer to the output layer can be considered as 2 projections; fixing one lens behind and adjusting only the first lens can achieve the same effect of adjusting 2 lenses at the same time.

$$
t_{e}=\sum_{h=1}^{H} w_{e h} p_{h m} \cdot z_{m} .
$$




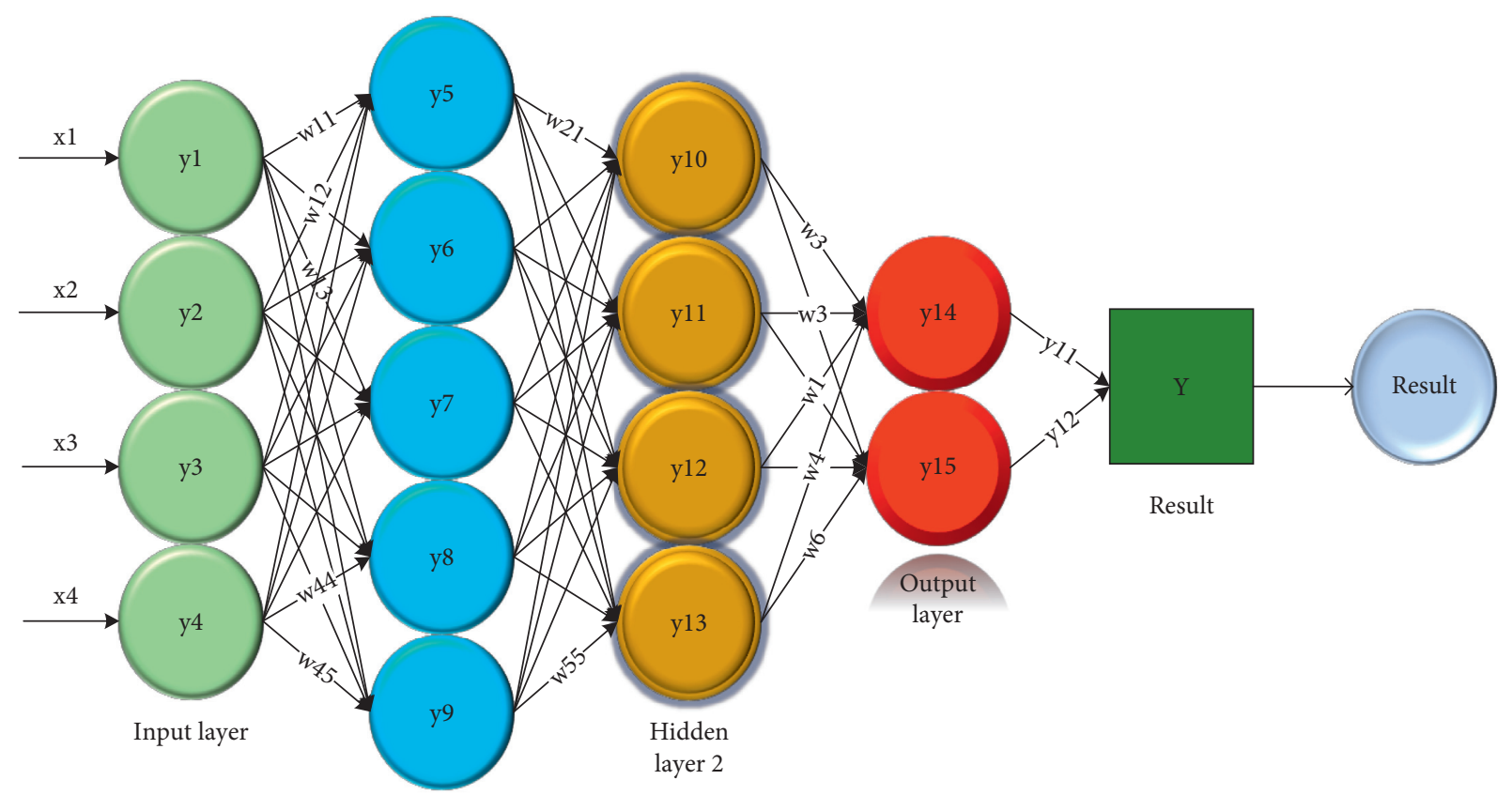

FIGURE 2: Sliding window recurrent neural network with fully connected dimensionality reduction layers.

Since this paper focuses on the classification problem, the following loss function is defined:

$$
\nabla F=\frac{1}{Q E} \sum_{q=1}^{Q} \sum_{e=1}^{E}\left(t_{e} \cdot t_{e 0}+g\right)^{2}
$$

In addition to the empirical risk minimization and structural risk minimization strategies, the MCNNA in this paper also introduces a core idea-design risk minimization, which can improve overfitting by combining design risk minimization with empirical risk minimization and structural risk minimization. This section focuses on theoretical aspects of design risk minimization. Since the design risk minimization involves the selection of optimal control parameters, we first introduce the structural risk, which is used to derive the concept of control parameters. Specifically, we consider the response of the $h$ th node of the hidden layer to the change of the $l$ th dimension and the $m$ th dimension of the input, and the corresponding second-order derivative is

$$
\begin{aligned}
\frac{\partial^{2} p_{h}}{\partial z_{l} \partial z_{m}} & =\beta_{h}^{2} v_{h l} f_{h}^{\prime \prime}\left(\beta_{h}^{2} k_{h}\right), \\
g & =\left(\frac{\partial L}{\partial y_{1}}, \frac{\partial L}{\partial y_{2}}, \ldots, \frac{\partial L}{\partial y_{k}}, \ldots\right) .
\end{aligned}
$$

If the current layer criterion function is

$$
L^{\prime}-y^{\prime} \otimes g
$$

the return of the gradient extraction function is

$$
\left(L^{\prime}, x\right)=\sum_{k}\left(g_{k} \cdot \frac{\left(\partial L / \partial y_{1}\right)}{\left(\partial L / \partial y_{2}\right)}\right)
$$

Then, the gradient of the previous layer is extracted and input to the network input node of the next layer; the gradient of the previous layer can be passed to the current layer by the function; here, the gradient extracted from the previous layer can be seen as the target vector of the criterion function. The main research focus of the paper is the longtime dependence problem, the layered sliding window recurrent neural network is the model structure used to solve this problem in this paper, and the key to the layered sliding window recurrent neural network to be able to work is to integrate the posterior semiaccumulative model or interval accumulation model in it. This dataset has only two sequences, and the elements in the sequence are real numbers instead of vectors, to speed up the calculation.

3.2. Experimental Design for the Distribution of the Benefits of Technological Innovation in Human Capital. The type of business ownership is another factor that may affect HRM activities and business performance. On the one hand, SOEs tend to provide employees with more employment security than non-SOEs; on the other hand, SOEs also give employees less discretionary opportunities, and they regulate their behavior mainly through rules and regulations, while many non-SOEs want to motivate their employees by increasing their autonomy and self-direction. First, although this method improves the discounted future income method for employee compensation accounting period which is default to retirement or death, using the employee's salary within the next five years, this method does not require the assumption that employees can work until retirement one by one to assess the value of human capital [19]. Although this minimizes the impact of early separation on the human capital value, we believe that it seems to underestimate the human capital value of employees to some extent, because 
this method continues the discounted future income method. We believe that the difference in the value of human capital is not solely due to the profitability of the firm and that using wages alone to determine the ability of employees to estimate their human capital value would be biased. Finally, in the process of discounting, the choice of the discount rate is based on our experience only, and there is no tested process of calculating the discount rate, while the value of human capital is constantly changing with the environment, and the interest rates of bank deposits are changing day by day, but the value of human capital should be more stable, contrary to this, so this improved model still has shortcomings, as shown in Figure 3.

The human capital theory is a part of human resource management theory, and according to modern human resource investment management theory, highly skilled personnel training is also an act of human capital investment. This act is mainly initiated by the state, schools, and enterprises. At the national level, it is a policy-oriented act to build up a pool of highly skilled talents, but it is a national act to manage the human resources of a country or a region. Only when the state makes corresponding plans from the macro policy level can the skills of skilled talents be applied, played, and promoted and the ladder of skilled talents be reasonable and balanced. Therefore, in the cultivation of highly skilled talents, the state's investment is the most basic and oriented. The mode of production presents a characteristic of intelligence. The emergence of this characteristic does not mean that highly skilled personnel are no longer needed to complete the corresponding front-line work, but that the advanced manufacturing industry needs top-notch technical personnel to give play to light and heat. The school is a public welfare and nonprofit unit, and for the cultivation of high-skilled talents, the investment in talents is mainly realized through the financial allocation of the government. Schools can initially decide the specifications and levels of skilled personnel training according to policies and regulations and realize the initial management of skilled personnel. Enterprises' management and investment in highly skilled talents are mainly manifested as an investment in their development, investment in enterprise technology, and investment in human resource training. As the medium and microelements of investment in high-skilled talents, enterprises are responsible for the task of growth and deployment of high-skilled talents. See Figure 3.

Forecasting is the use of information, knowledge, and tools that people already must predict and judge the future trend and outcome of things [20]. Forecasting has been around since ancient times, but it has only been a selfcontained discipline for a few decades. From the perspective of forecasting theory, the conventional methods of forecasting are qualitative forecasting method, constraint extrapolation, and simulation model forecasting method. Qualitative forecasting methods mainly include expert forecasting, Delphi forecasting, subjective probability forecasting, and cross-probability forecasting. However, the qualitative forecasting method is more subjective and subject to more uncontrollable factors, so it is mostly used as an auxiliary forecast. The constraint extrapolation method mainly includes trend extrapolation, iterative extrapolation, etc., and is mostly used for time series forecasting. The simulation model forecasting method refers to the establishment of one or several mathematical models, which is suitable for non-time series forecasting, and the main methods include regression analysis, correlation analysis, and elasticity coefficient method [21-23].

The application of artificial intelligence in the field of computer vision is mainly divided into three stages, such as detecting the object, recognizing the object, and the purpose of recognizing the object. The current application of artificial intelligence in the field of computer vision is more common in three directions, such as face recognition, pupil recognition, and fingerprint recognition. High-skilled talents reupdate technology and recreate products, thereby driving the improvement of enterprise's total factor productivity and accelerating the pace of industrial structure transformation and upgrading. In addition, artificial intelligence in the field of computer vision object scene recognition technology is not mature enough. This is because the type of recognition target is complex and variable. Although the use of artificial intelligence to recognize the appearance of the target characteristics has still a certain error rate, the application of artificial intelligence in the field of computer vision still needs to be explored deeply, to improve the accuracy and precision of pattern recognition technology, as shown in Figure 4.

With theoretical and methodological advances in management and organizational behavior research, an increasing number of studies have integrated macro- and microperspectives to detect how multilevel factors affect outcome variables at different levels of analysis. Psychological capital has been supported by studies that directly influence leaders' and employees' work behavior, work attitudes and performance, and in turn, overall firm performance. However, little research has been conducted on psychological capital and firm technological innovation performance, especially examining the relationship between psychological capital, organizational innovation climate, and firm technological innovation performance from a crosslevel perspective. This study categorizes psychological capital and technological innovation performance as individuallevel factors and analyzes organizational innovation climate as a group-level factor in a cross-level analysis. The main purpose of this study is to further test the results of the empirical study in the previous chapter and to focus on the moderating effect of organizational innovation climate on psychological capital and technological innovation performance.

\section{Analysis of Results}

4.1. Results of the Hierarchical Neural Network Power Source Model. The window model in the hierarchical sliding window recurrent neural network is the interval accumulation model, the recurrent neural network is GRU, the hidden layer vector length of GRU is 200, the activation function is the rectified linear unit activation function, the window width is 33 , and the span step length is 16 . The 


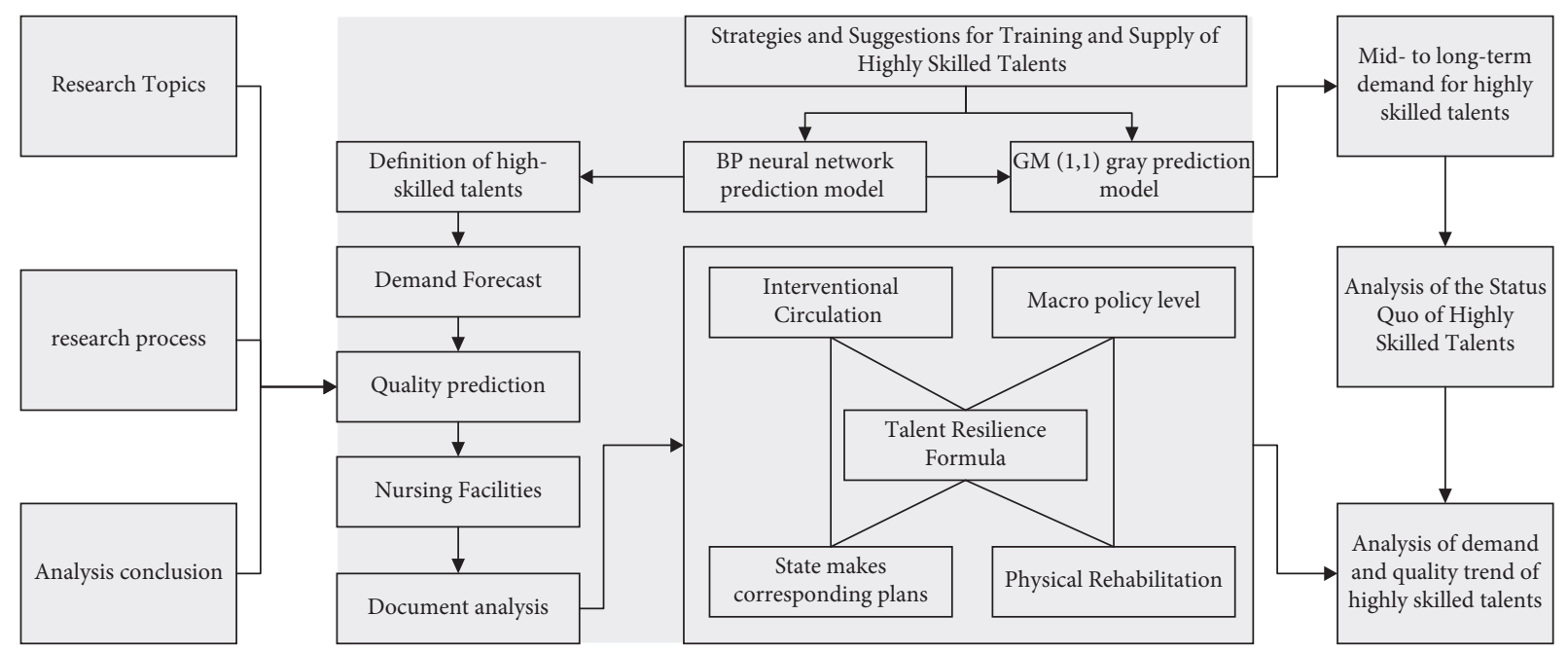

FIGURE 3: Technology roadmap.

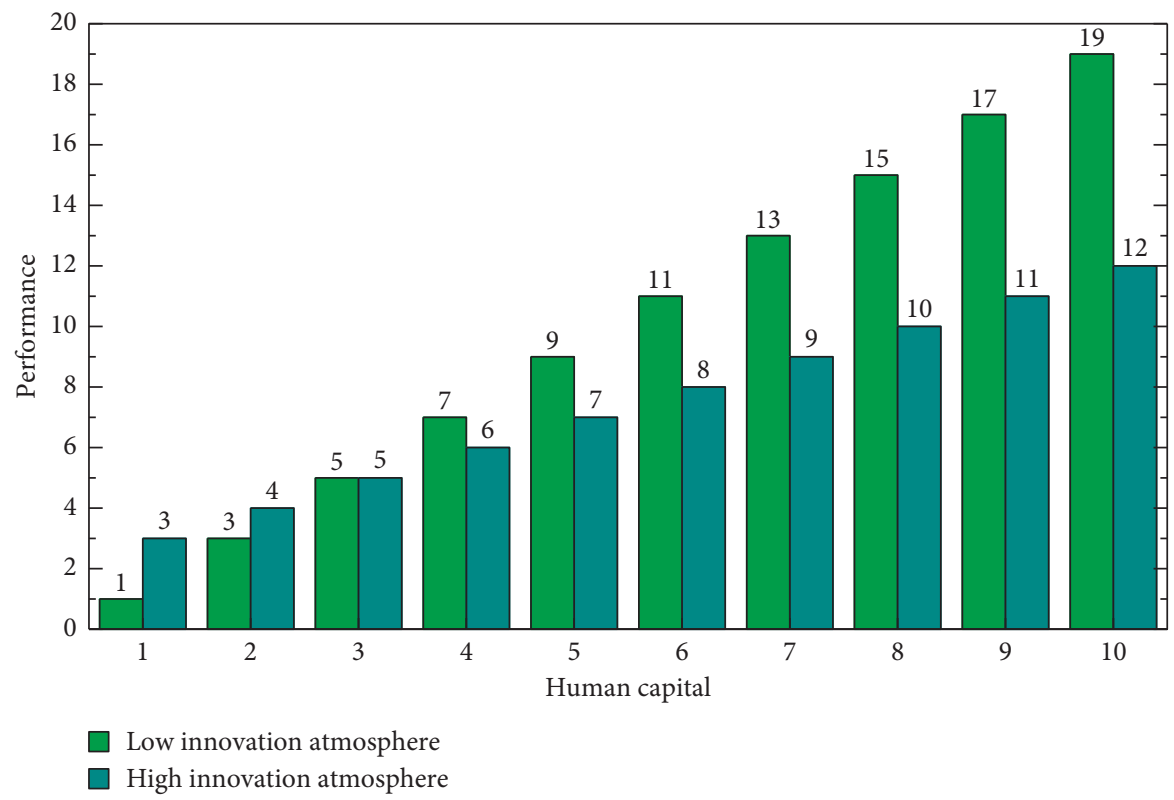

FIGURE 4: Interaction between psychological capital and organizational innovation climate on firms' technological innovation performance.

weight update optimization scheme is the adaptive impulse estimation algorithm (Adam), the learning step length is 0.0001 , and the classifier is Softmax. The experimental results are shown in Figure 5.

In this paper, we also test the convergence ability of the full-sequence cumulative model, where the recurrent neural network in the hierarchical sliding window recurrent neural network is GRU, the hidden layer vector length of GRU is 200 , the activation function is rectified linear unit activation function, the window width is 33 , and the span step length is 16. The weight update optimization scheme is the adaptive impulse estimation algorithm (Adam), the learning step length is 0.0001 , the classifier is Softmax classifier, the criterion function is cross-entropy criterion function, and the dropout parameter is 1 . The effect of human capital on enterprise technological innovation performance is not sufficient, and it is necessary to find other elements to give full play to the utility of human capital. The training error is 0.693 after 10,000 iterations on the dataset $\mathrm{A}$ with a sequence length of 1800 .

The number of samples in each batch of batch gradient descent is 50, the recurrent neural network in the hierarchical sliding window recurrent neural network is GRU neural network, the vector length of the hidden layer of GRU is 512 , the activation function is rectified linear unit activation function, the window width is 33 , the span step length is 8 when there is no fully connected dropout layer, and the span step length is 2 when there is a fully connected dropout layer. The weight update optimization scheme is adaptive impulse estimation algorithm (Adam), the learning step is 0.00001, the classifier is Softmax classifier, the criterion function is cross-entropy criterion function, the dropout is 


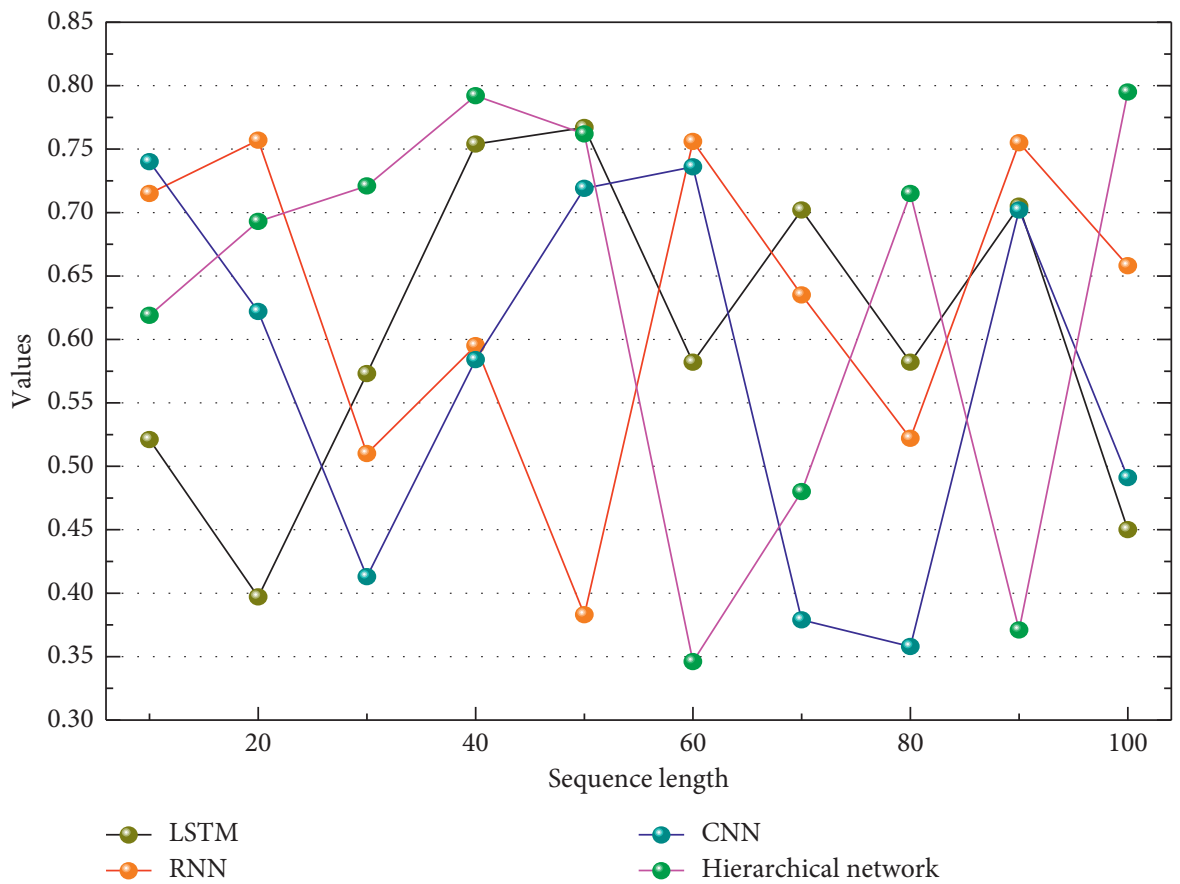

Figure 5: Training errors of the two models after 10,000 iterations on dataset A with different sequence lengths.

performed for the output layer of each layer, the dropout parameter is 0.1 in the training phase, that is, $90 \%$ of the neurons are randomly selected in the output vector of each layer so that the value and gradient of these neurons are zero, and the dropout parameter is 1 in the testing phase; that is, no dropout is performed. The parameter is 1 , i.e., no dropout, and all other parameters are the default values provided by TensorFlow.

In each training period, the entire training set is traversed, the order of the training set is randomly disrupted before each training period, and the correctness of the validation set is calculated after each training period. Before starting training, define a Python variable that holds the "maximum correctness of the validation set" and initialize it to 0 . If the correctness of the current validation set is greater than the "maximum correctness of the validation set," the correctness of the current validation set is saved to the variable representing. If the correctness of the current validation set is greater than the "maximum correctness of the validation set," the correctness of the current validation set is saved to the variable representing the "maximum correctness of the validation set" and the current model is saved to the "model and weight coefficient file." After enough training periods, 3000 in this paper, the saved model is run in the test set to obtain the correctness of the test set, as shown in Figure 6.

The correctness of the test set of the no optical flow model of the long-time recurrent convolutional network on the split01 splitting scheme of UCF101 is $71.12 \%$, while the correctness of the test set of the interval accumulation model of the hierarchical sliding window recurrent neural network without fully connected reduced layers on the split01 splitting scheme of UCF101 is $80.20 \%$, so the hierarchical sliding window recurrent neural network is more effective than the long-time recurrent convolutional network with no optical flow model, indicating that the ability of the hierarchical sliding window recurrent neural network to alleviate the long-time dependence problem in real scenarios is effective. The difference in the correctness between the test set of the hierarchical sliding window recurrent neural network with and without the fully connected descending layer model on both the KTH dataset and the UCF101 dataset is not significant, while the fully connected descending layer takes up computational resources, so the hierarchical sliding window recurrent neural network without the fully connected descending layer is more efficient than the hierarchical sliding window recurrent neural network with the fully connected descending layer. In practical applications, the model without fully connected descending layers should be preferred.

4.2. Results of Human Capital Innovation Benefit Distribution. First, the development of intelligence cannot be separated from the support of the Internet, big data, and cloud computing, so the Internet penetration rate is chosen to measure the level of intelligence. Secondly, since the industrial field is currently the main application area of AI technology, and industrial robots are also the key to the profound integration of AI technology and industrialization, the development level of industrial robots is used to measure the development and application of AI, and the sales volume of industrial robots is chosen to quantify correspondingly. Although the number of graduates from higher vocational colleges and universities has increased, the proportion of graduates who have obtained advanced certificates has 


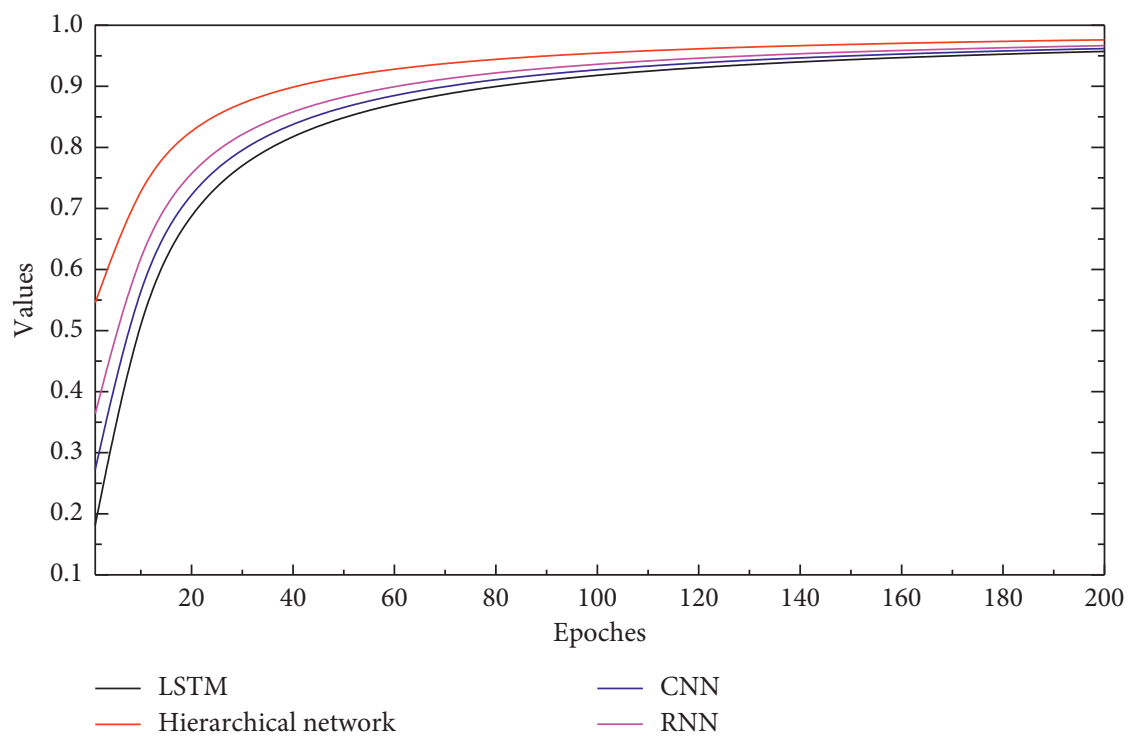

Figure 6: Correct rate.

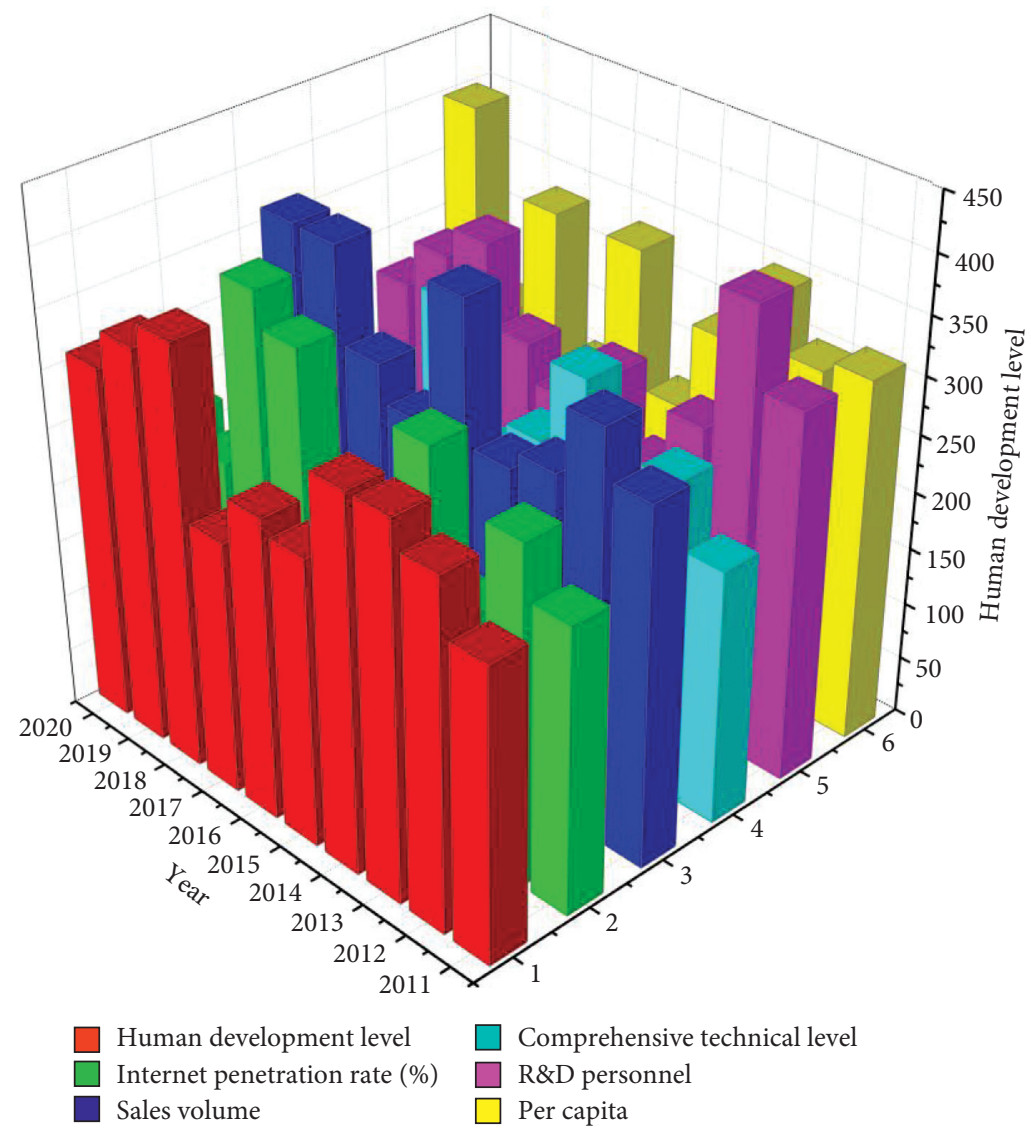

FIgURE 7: Technological progress, capital investment, and human development.

shown a decreasing trend, which shows that few students among graduates can directly transform their academic qualifications into skills and vocational qualifications. As a base for the growth of highly skilled talents, the growth and cultivation of skilled talents are still lacking, which leads to the embarrassing situation that some students enter the society without a certificate in their hands after graduation and intensifies the imbalance between the supply of highly skilled talents and the demand of social enterprises, as shown in Figure 7. 


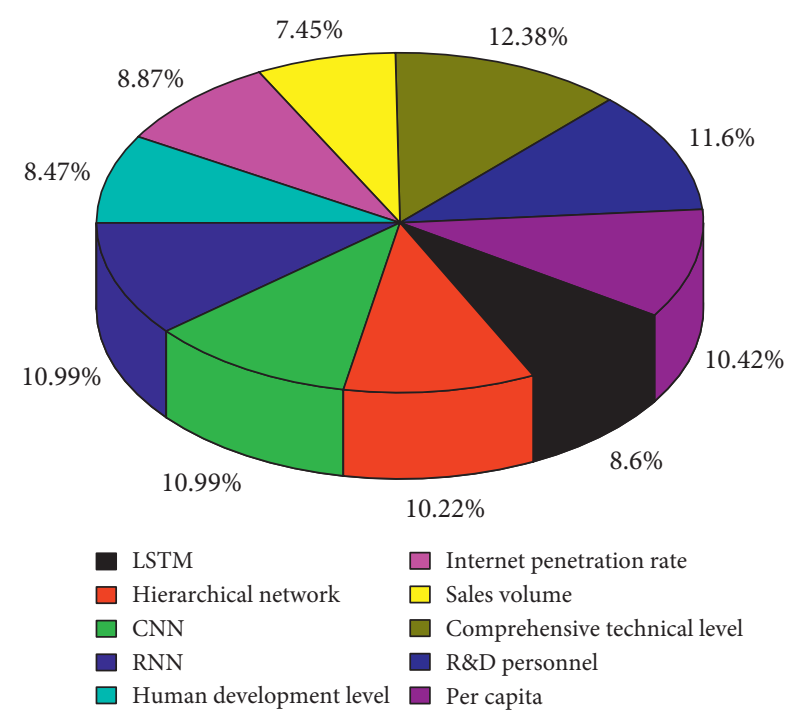

Figure 8: Number of technical staff.

Construct validity includes convergent validity and discriminant validity. Construct validity has been tested in previous studies through exploratory factor analysis. If common factors can be effectively extracted from the exploratory factor analysis and these factors are close to the theoretical structure, then the instrument can be considered to have construct validity. However, the use of exploratory factor analysis to test construct validity is controversial, and one of the major drawbacks is that theoretical roles are determined ex-post rather than ex-ante, which makes the constructs obtained from exploratory factor analysis more statistical than logical. As a result, academics are gradually using validated factor analysis, which is both logical and operational, to conduct tests of convergent and discriminant validity, and so on until the length of the final sequence is within the allowable range of the cyclic neural network, forming a layered structure composed of multiple cyclic neural networks.

In most empirical studies, we need to standardize the available data to eliminate the effect of standardized magnitudes of indicators. The unit and value gaps between indicators make this step increasingly necessary. Instead of standardization, we process the raw data to obtain the deviation of an indicator from the mean of all samples. Since both qualitative and quantitative indicators exist, qualitative indicators should be quantified first and then standardized; quantitative indicators are difficult to compare between indicators due to different units, and the second is the large difference in values, so they should be standardized. The comparability of the data after the standardization process is enhanced, as shown in Figure 8.

Finally, for the unobtained market survey questionnaire data, it is not known whether there is consistency in the results and whether there is reasonableness and realism in the determination of the indicator weights. In the process of applying mathematics and statistics, systematic errors are inevitable, and if the uncontrollable systematic errors are too large in our study, they directly affect the results of the experiment. However, based on the unrealistic nature of a comprehensive survey, one can only accept the impact of systematic errors on the experimental results.

\section{Conclusion}

Considering that the difference between the full-sequence accumulation model and the post-half accumulation model lies in the ratio of the number of elements accumulated to the window width, this paper designs the interval accumulation model, which adds the element vectors at the odd moments in the RNN output sequence within the window. The difference in accuracy between the semiaccumulative model and the interval-accumulative model is not significant, indicating that the number of element vectors added rather than the position is the factor affecting the effectiveness of the model. Consider that the difference between the full-sequence accumulation model and the latter half accumulation model is mainly the ratio of the accumulated vector elements to the vector elements of the entire window. And the test accuracy of the two semiaccumulative models is more than $8 \%$ higher than that of the long-time recurrent convolutional network with no optical flow model. The positive effect of bundling is also validated for the combination of HR practices. First, we find that motivation alone can have a significant negative effect on turnover, but it must be bundled with competency to significantly increase organizational performance. Next, the improvement of organizational performance relies on both competency development, which ensures that the level of human capital in the organization meets the requirements for achieving strategic goals, and motivation, which motivates employees to work, and their interaction has a significant positive impact on organizational performance.

\section{Data Availability}

The data used to support the findings of this study are included within the article.

\section{Conflicts of Interest}

The authors declare no conflicts of interest.

\section{References}

[1] G. Yang, X. Zhou, and Z. Liang, "Enterprise internationalisation performance evaluation model based on artificial neural network," International Journal of Embedded Systems, vol. 13, no. 4, pp. 387-397, 2020.

[2] S. M. H. Bamakan, N. Faregh, and A. ZareRavasan, "DiANFIS: an integrated blockchain-IoT-big data-enabled framework for evaluating service supply chain performance," Journal of Computational Design and Engineering, vol. 8, no. 2, pp. 676-690, 2021.

[3] A. Protogerou, S. Papadopoulos, A. Drosou, D. Tzovaras, and I. Refanidis, "A graph neural network method for distributed anomaly detection in IoT," Evolving Systems, vol. 12, no. 1, pp. 19-36, 2021. 
[4] H. Ma, X. Zhang, F. Ju, and S.-B. Tsai, "A study on curing kinetics of nano-phase modified epoxy resin," Scientific Reports, vol. 8, no. 1, p. 3045, 2018.

[5] S. Smetana, K. Aganovic, and V. Heinz, "Food supply chains as cyber-physical systems: a path for more sustainable personalized nutrition," Food Engineering Reviews, vol. 13, no. 1, pp. 92-103, 2021.

[6] D. Pattanaik, S. Mishra, G. P. Khuntia, R. Dash, and S. C. Swain, "An innovative learning approach for solar power forecasting using genetic algorithm and artificial neural network," Open Engineering, vol. 10, no. 1, pp. 630-641, 2020.

[7] R. Wiedmer and S. E. Griffis, "Structural characteristics of complex supply chain networks," Journal of Business Logistics, vol. 42, no. 2, pp. 264-290, 2021.

[8] X. Xiao-wei, "Study on the intelligent system of sports culture centers by combining machine learning with big data," Personal and Ubiquitous Computing, vol. 24, no. 1, pp. 151-163, 2020.

[9] Z. L. Yang, N. Islam, Y. Shi, K. Venkatachalam, and L. Huang, "The evolution of interindustry technology linkage topics and its analysis framework in 3D printing technology," IEEE Transactions on Engineering Management, vol. 1, pp. 1-21, 2021.

[10] E. H. Özder, E. Özcan, and T. Eren, "Sustainable personnel scheduling supported by an artificial neural network model in a natural gas combined cycle power plant," International Journal of Energy Research, vol. 44, no. 9, pp. 7525-7547, 2020.

[11] A. K. Ghadiyaram, "A compendium on edge computing where Ai can meet," Turkish Journal of Computer and Mathematics Education, vol. 12, no. 10, pp. 4118-4126, 2021.

[12] L. Zhou, "Product advertising recommendation in e-commerce based on deep learning and distributed expression," Electronic Commerce Research, vol. 20, no. 2, pp. 321-342, 2020.

[13] C. Giudicianni, M. Herrera, A. d. Nardo, K. Adeyeye, and H. M. Ramos, "Overview of energy management and leakage control systems for smart water grids and digital water," Modelling, vol. 1, no. 2, pp. 134-155, 2020.

[14] Y. Ding, W. Jiang, Q. Lou et al., "Hardware design and the competency awareness of a neural network," Nature Electronics, vol. 3, no. 9, pp. 514-523, 2020.

[15] K. Migdał-Najman, K. Najman, and S. Badowska, "The GNG neural network in analyzing consumer behaviour patterns: empirical research on a purchasing behaviour processes realized by the elderly consumers," Advances in Data Analysis and Classification, vol. 14, no. 4, pp. 947-982, 2020.

[16] D. Gao, Y. Liu, Z. Guo et al., "A study on optimization of CBM water drainage by well-test deconvolution in the early development stage," Water, vol. 10, no. 7, 2018.

[17] Z. Yang, W. Zhang, F. Yuan, and N. Islam, "Measuring topic network centrality for identifying technology and technological development in online communities," Technological Forecasting and Social Change, vol. 167, Article ID 120673, 2021.

[18] K. Demertzis, L. Iliadis, N. Tziritas, and P. Kikiras, “Anomaly detection via blockchained deep learning smart contracts in industry 4.0," Neural Computing and Applications, vol. 32, no. 23, pp. 17361-17378, 2020.

[19] C. Liu and X. Zheng, "Exploring resource management for innovation power network based on deep learning algorithm," Neural Computing and Applications, vol. 33, no. 9, pp. 4013-4025, 2021.

[20] C. Hongfeng, "Information network security construction based on depth learning and modulus algorithm," Journal of
Intelligent and Fuzzy Systems, vol. 38, no. 6, pp. 7229-7240, 2020.

[21] T. Grubljesic, P. S. Coelho, and J. Jaklic, "The shift to socioorganizational drivers of business intelligence and analytics acceptance," Journal of Organizational and End User Computing, vol. 31, no. 2, pp. 37-64, 2019.

[22] L. Z. Zhang, M. Mouritsen, and J. R. Miller, "Role of perceived value in acceptance of "Bring your own device" policy," Journal of Organizational and End User Computing, vol. 31, no. 2, pp. 65-82, 2019.

[23] A. Shahri, M. Hosseini, K. Phalp, J. Taylor, and R. Ali, "How to engineer gamification," Journal of Organizational and End User Computing, vol. 31, no. 1, pp. 39-60, 2019. 